\title{
Gradient analysis of soil-plant interactions from the alpine-nival ecotone to the snowline on slopes of the Central Great Caucasus (Kazbegi Region, Georgia)
}

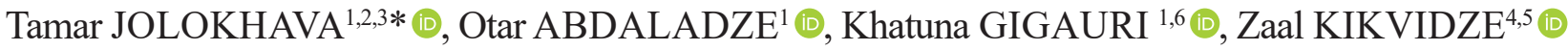 \\ ${ }^{1}$ School of Natural Sciences and Medicine, Institute of Ecology, Ilia State University, K. Cholokashvili Ave. 3/5, Tbilisi 0162, Georgia \\ ${ }^{2}$ Faculty of Exact and Natural Sciences, Ivane Javakhishvili Tbilisi State University, I. Chavchavadze Ave. 3, Tbilisi 0179, Georgia \\ ${ }^{3}$ Ministry of Environmental Protection and Agriculture of Georgia, Science-Research Centre of Agriculture, Soil Fertility Division, \\ Marshal Gelovani Ave. 6, Tbilisi 0160, Georgia \\ ${ }^{4}$ Institute of Ethnobiology and Socio-ecology, Ilia State University, K. Cholokashvili Ave. 3/5, Tbilisi 0162, Georgia \\ ${ }^{5}$ Institute of Botany, Ilia State University, Botanikuri Str. 1, Tbilisi 0105, Georgia \\ ${ }^{6}$ Georgian Institute of Public Affairs, School of Government, Ietim Gurji Str. 9, Tbilisi 0105, Georgia
}

\begin{abstract}
Alpine ecosystems are especially sensitive to climatic changes which affect the relationships among glaciers, snow, vegetation and soils. Our aim was to examine how the variation in the abiotic environment affected soil properties and plant species distribution at regional and local scales. We sampled soil and vegetation along two transects set on the opposite-facing slopes (North versus South), from the alpine-nival ecotone to the snowline (Central Great Caucasus, Kazbegi, Georgia). We measured also soil temperature and controlled for the slope inclination. Multivariate ordination methods were used to link abiotic factors, soil properties and plant species distribution along the gradients. We found that ordination models were better resolved when soil properties were used as environmental variables instead of abiotic ones such as elevation, inclination and slope aspect. Soil $\mathrm{pH}$ and plant available potassium were the best predictors of plant species distribution in these habitats. We conclude that the models that account for the role of soils as a mediator between the abiotic environment and vegetation can more accurately describe plant species distribution at local and regional scales: a potentially important amendment with implications for the monitoring of the effects of climate change on vegetation at least in high mountain systems.
\end{abstract}

Keywords: abiotic variables, elevation gradient, soil properties, species composition, subnival-nival zone

Article history. Submitted 09 February 2021. Revised 24 May 2021. Published 29 June 2021

Citation. Jolokhava T., Abdaladze O., Gigauri K., Kikvidze Z. 2021. Gradient analysis of soil-plant interactions from the alpine-nival ecotone to the snowline on slopes of the Central Great Caucasus (Kazbegi Region, Georgia). Ukrainian Botanical Journal, 78(3): 163-175. https://doi.org/10/15407/ukrbotj78.03.163

*Corresponding author (e-mail: tamar.jolokhava.1@iliauni.edu.ge)

(C) 2021 T. Jolokhava, O. Abdaladze, K. Gigauri, Z. Kikvidze. Published by the M.G. Kholodny Institute of Botany, NAS of Ukraine. This is an open access article under the terms of the Creative Commons Attribution License (http://creativecommons.org/licenses/by/4.0/), which permits use, distribution, and reproduction in any medium, provided the original work is properly cited 


\section{Introduction}

Soils are important medium which can connect vegetation patterns with ecological processes (Chapin et al., 1995; Dahlgren, 2006; Budzhak, Didukh, 2020). In mountains, where easily discernible gradients were instrumental for studying plant species distributions (Lomolino, 2001; Körner, 2007), clear differences were documented in these distribution patterns between calcareous and siliceous soils (e.g., Michalet et al., 2002; see Tudela-Isanta et al., 2018 for the recent contribution); the importance of soil physical and chemical properties to plant life were also emphasized (Tale, Ingole, 2015; Praeg et al., 2019). The fact that soil formation is influenced by plants and not only by climate, geology, topography and time (Jenny, 1994), and that plants can affect soil processes (Gleason, 1926; Hooper et al., 2000; Eisenhauer et al., 2013) is well appreciated, for example in the studies on successions (Kardol et al., 2006). However, in the descriptions and analyses of vegetation on ecological gradients, soils are usually treated as a part of environment which participates in developing vegetation patterns through ecophysiological performance and microbiota (Körner, 2003; Molau et al., 2005; Devictor et al., 2010; Donhauser, Frey, 2018), and the feedback between vegetation and soil is rarely analysed in the context of species spatial distributions. Conversely, the variation in the abiotic environment on large temporal and spatial scales is considered to be an excellent predictor of plant community composition (Huston, Huston, 1994), even though the inclusion of soil variables in the set of environmental variables improves predictive power of the models based on abiotic variation (Buri et al., 2020). Therefore, a question arises as to whether an important knowledge can be gained by explicitly including the relations between, on the one hand, abiotic variables and soil, and, on the other hand, soil and plants, in the analyses? Addressing this question was the general goal of our study.

The existing knowledge on the interrelations among abiotic variables, soil properties and plant growth can be depicted as a three-component process (Fig. 1). The first component is abiotic variables (temperature, precipitation, topography, bedrock type), which vary independently from the other components but affect both soil formation and vegetation growth. The second component is soil, which depends greatly on the first component. At the same time, soils exert important influence on the abundance and species composition of plant cover and thus can act as a intermediary link between abiotic variables and vegetation. Finally, the

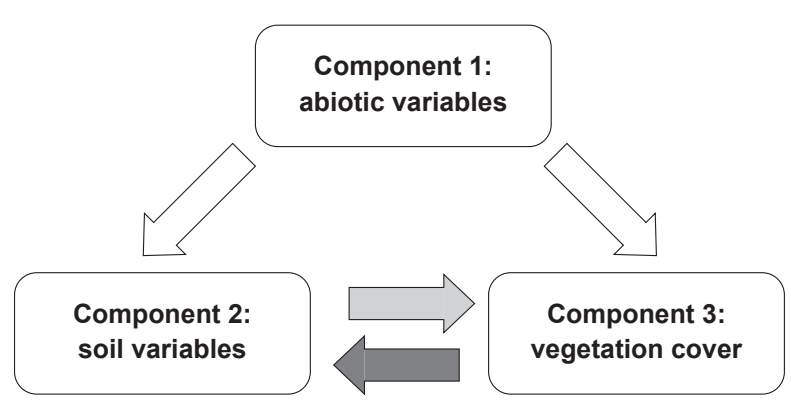

Fig. 1. A scheme of relationships among the abiotic environment, soil properties and vegetation. Abiotic variation affects soil and vegetation (white arrows), while vegetation depend on soil properties. The relationship between soil and vegetation is reciprocal: soil properties affect the abundance and species composition of the vegetation (light gray arrow), while plant processes affect soil development (dark gray arrow).

third component is vegetation, which directly depends on the first component, but is also influenced by abiotic variables indirectly via the soil properties; at the same time, vegetation processes feed back and can be an important contributor to soil formation, for example, via accumulation of organic matter (Bezemer et al., 2006; Brevik, 2013). Such a scheme enables us to perform a two-step analysis of the importance of soil properties to vegetation patterns, in which soils are treated not as a part of environment for plants but as a function dependent on abiotic variables. The same methods of multivariate analysis employed for vegetation analyses, e.g., Principal Component Analysis (PCA) or non-Metric Dimensional Scaling (nMDS), might be used at the first step. The obtained model that describes how the soil properties ( $\mathrm{pH}$, nutrient, organic matter, temperature) change along abiotic gradient can then be linked to species distributions on the second stage using again multivariate ordination method such as Canonical Correspondence Analysis (CCA). For example, multivariate analyses can produce "components" or "coordinates" that involve collinear variables and help incorporate a bulk of abiotic variation along ecological gradients (Palmer, 2004). Further, when combined with correlation analyses, these composite factors can help identify the best predictors of plant distribution along the gradient. We hypothesized that, because soil is directly linked to plants, an ordination based on soil variables will be better resolved than the one based on abiotic variables. For this purpose we employed the CCA, in which we used four sets of environmental variables: (1) two coordinates obtained from nMDS, (2) two components from PCA, (3) two soil variables selected as best predictors after the nMDS and (4) two soil variables selected as best predictors after the PCA. Statistical significance and eigenvalues were used 
to evaluate the success of ordination in each case. Our primary expectation was that ordinations based on soil variables would be better resolved than those based on abiotic variables, which would be an indication of the importance of soil properties to plant species distribution on ecological gradients. Our secondary aim was to see which variables would appear to be the best predictors of plant species distributions in the settings of our case study, which we conducted in the Central Great Caucasus mountains along an elevation gradient on the slopes of Mt. Kazbegi, in the subnival-nival belt from the alpinenival ecotone to the snowline: a prominent habitat with very clear gradients of environmental conditions related to elevation (Körner, Paulsen, 2017; Nakhutsrishvili, Abdaladze, 2017a).

\section{Materials and Methods}

\section{Study area}

Our study was conducted on the slopes of Mt. Kazbegi in the Central Great Caucasus Mountains (Fig. 2). The exact coordinates for the lowest and highest points of the transect are as follows: the north slope - from

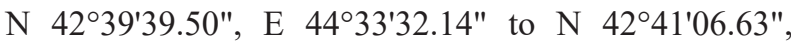

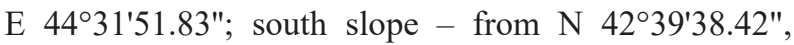

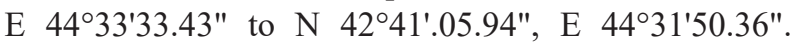
Deposits of the Jurassic period, in particular Lias (Lower Jurassic), are most widespread in the region. The rocks are composed of slates and quartzite. Sandy loams and diabasic veins are also found. The topography is characterised by high bare sharp-ridged rocky ranges divided by large depressions and narrow ravines of erosive and tectonic origin (Nakhutsrishvili, 1998; Abdaladze et al., 2015). Kazbegi municipality area is $1081.7 \mathrm{~km}^{2}$, the alpine-nival ecotone is located at ca. $3000 \mathrm{~m}$ a.s.1., and the snowline runs at ca. $3950 \mathrm{~m}$ a.s.1. The lowest, average and highest elevations of the region are $1210 \mathrm{~m}$ a.s.1., $2850 \mathrm{~m}$ a.s.1. and $5033 \mathrm{~m}$ (Maruashvili, 1971; Nakhutsrishvili et al., 2005). The following factors

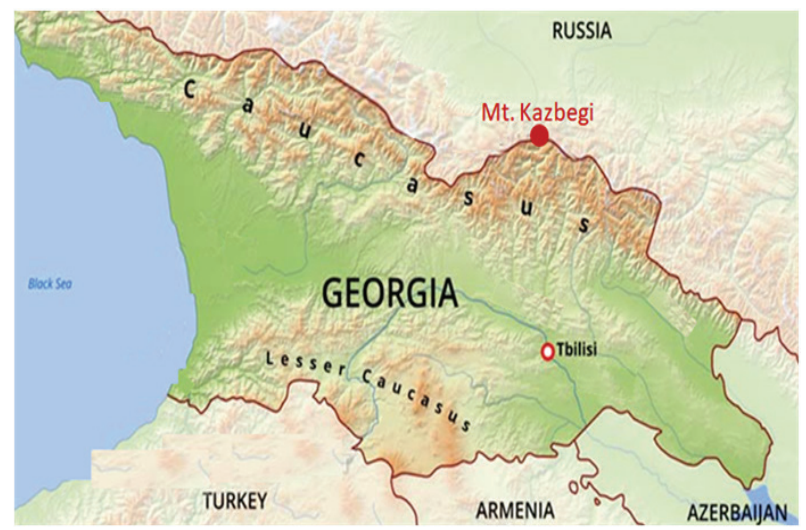

Fig. 2. Location of the Kazbegi Region, the Central Great Caucasus (marked). (N 42³9'; E 4437')

determine the climate in the region: high elevations, complex topography, and location on north-facing macro-slope of the Great Caucasus. Table 1 represents some main characteristics of climate of the region (Nakhutsrishvili, 2003; Abdaladze et al., 2015). The soil cover mainly consists of montane forest-meadow and montane meadow soils of more than ten types, subtypes and genera (Talakhadze et al., 1985; Urushadze, 1989). The vegetation of the alpine desert is represented by vegetation patches, where species aggregate in clumps; the patches are also formed by the growth forms adapted to the harsh conditions of high elevations: cushions, prostrate plants, mound-building plants, etc. (Körner, Larcher, 1988; Kikvidze, 1993; Kikvidze, Nakhutsrishvili, 1998; Körner, 2011; Nakhutsrishvili, Abdaladze, 2017b). The study area is characterized by high richness of the Caucasus endemic species (26\%) and genera (6 out of 11) (Kharadze, 1965; Nakhutsrishvili, 2012; Solomon et al., 2014), as well as high diversity of plant communities (Nakhutsrishvili et al., 2005, 2006). The vegetation in the Central Caucasus mountains can be clearly divided into four zones: subalpine $(1800 / 1850$ 2450/2500 m a.s.1.), alpine (2450/2500-2950/3000 m a.s.1.), subnival (2950/3000-3650/3700 $\mathrm{m}$ a.s.1.) and nival (> $3700 \mathrm{~m}$ a.s.1.) (Nakhutsrishvili, 2003, 2012).

Table 1. Climate characteristics of the Kazbegi Region (according to: Nakhutsrishvili, 2003; Abdaladze et al., 2015)

\begin{tabular}{|c|c|c|c|c|c|c|c|}
\hline \multirow{3}{*}{ Zone } & \multicolumn{2}{|c|}{$\begin{array}{l}\text { Air temperature of warmest } \\
\text { months (July-August) }\end{array}$} & \multicolumn{2}{|c|}{$\begin{array}{l}\text { Air temperature of coldest months } \\
\text { (January-February) }\end{array}$} & $\begin{array}{l}\text { Duration of } \\
\text { snow cover }\end{array}$ & $\begin{array}{l}\text { Max. depth of } \\
\text { snow cover }\end{array}$ & $\begin{array}{l}\text { Ave. annual } \\
\text { precipitation }\end{array}$ \\
\hline & \multicolumn{2}{|c|}{${ }^{\circ} \mathrm{C}$} & \multicolumn{2}{|c|}{${ }^{\circ} \mathrm{C}$} & \multirow{2}{*}{ month } & \multirow{2}{*}{$\mathrm{cm}$} & \multirow{2}{*}{$\mathrm{mm}$} \\
\hline & Ave. & Abs. max. & Ave. & Abs. min. & & & \\
\hline Subnival & 10.0 & 31.0 & -12.0 & -30.0 & $7-8$ & $<200$ & $800-1000$ \\
\hline Lower part of nival zone & 2.2 & 15.0 & -14.0 & -33.0 & 12 & $>200$ & $1000-1200$ \\
\hline
\end{tabular}




\section{Sampling design}

The transect was set along a ridge where the slopes have well-pronounced $\mathrm{N}$ and $\mathrm{S}$ aspects. The study sites were established on two slopes of these opposite aspects from 3000 to $3900 \mathrm{~m}$ a.s.l. with an interval of $100 \mathrm{~m}$ a.s.l. (no sampling was conducted at $3400 \mathrm{~m}$ and 3500 $\mathrm{m}$ because the transect here was crossed by the glacier; $3800 \mathrm{~m}$ also appeared to be covered entirely with ice and sampling at this elevation was not possible). The given range transects the subnival and nival belts from alpinenival ecotone to the snowline (Fig. 3). Elevation and slope aspect were measured using a GPS device (Etrex Summit, Garmin, Switzerland). At each site, we randomly placed 20 sampling plots of $1 \mathrm{~m} \times 1 \mathrm{~m}$ size, therefore, we sampled $280 \mathrm{~m}^{2}$ area ( 7 elevations $\times 2$ slopes $\times 20$ plots). The distance between the plots was at least $4-5 \mathrm{~m}$. We measured slope inclination at each site using rulers and recorded it in degrees. Within each plot, all plant species were recorded, their abundance was measured by the frequency of occurrence, and vegetation projective cover (in \%) was estimated by visual assessment (Kent, 2011). For plant species names we followed the International Plant Names Index (IPNI). Soil samples were taken at a depth of 0-20 cm using a auger (Tan, 1995). From each quadrat, five samples of soil were taken from five randomly chosen points and mixed. The mixed samples $(7$ elevations $\times 2$ expositions $=14)$ were collected in paper bags and transferred to the laboratory. From each mixed sample, two replicates were analysed chemically: the samples were oven-dried at $35^{\circ} \mathrm{C}$, ground and sieved through a 2-mm sieve. Soil $\mathrm{pH}$ was determined in a 1:2.5 soil/water suspension using a $\mathrm{pH}$ meter (WTW Benchtop pH Meter InoLab® Multi 9310 IDS, Xylem Analytics, Germany) with a glass electrode (Jackson, 2005). Soil organic matter (SOM) was determined by Loss-onIgnition (LoI) with approximately $10 \mathrm{~g}$ of air-dry soil samples, using a high-temperature muffle furnace at temperatures of 105 and $360^{\circ} \mathrm{C}$; LoI organic matter is estimated by the difference in sample weight obtained at the above indicated temperatures (Schulte, Hopkins, 1996; Gavlak et al., 2003). Mobile forms of nitrogen were measured in potassium chloride extracts (Norman, Stucki, 1981), followed by ultraviolet spectrophotometry. Plant- available phosphorus was extracted with a sodium bicarbonate solution as described by Olsen (1954) with final photometric determination using the molybdenum blue method (Pansu, Gautheyrou, 2007) on a UV/VIS spectrophotometer (Specord 210 Plus, Analytik Jena, Germany). Plant-available potassium was determined in an ammonium nitrate extract (Stuanes et al., 1984) using
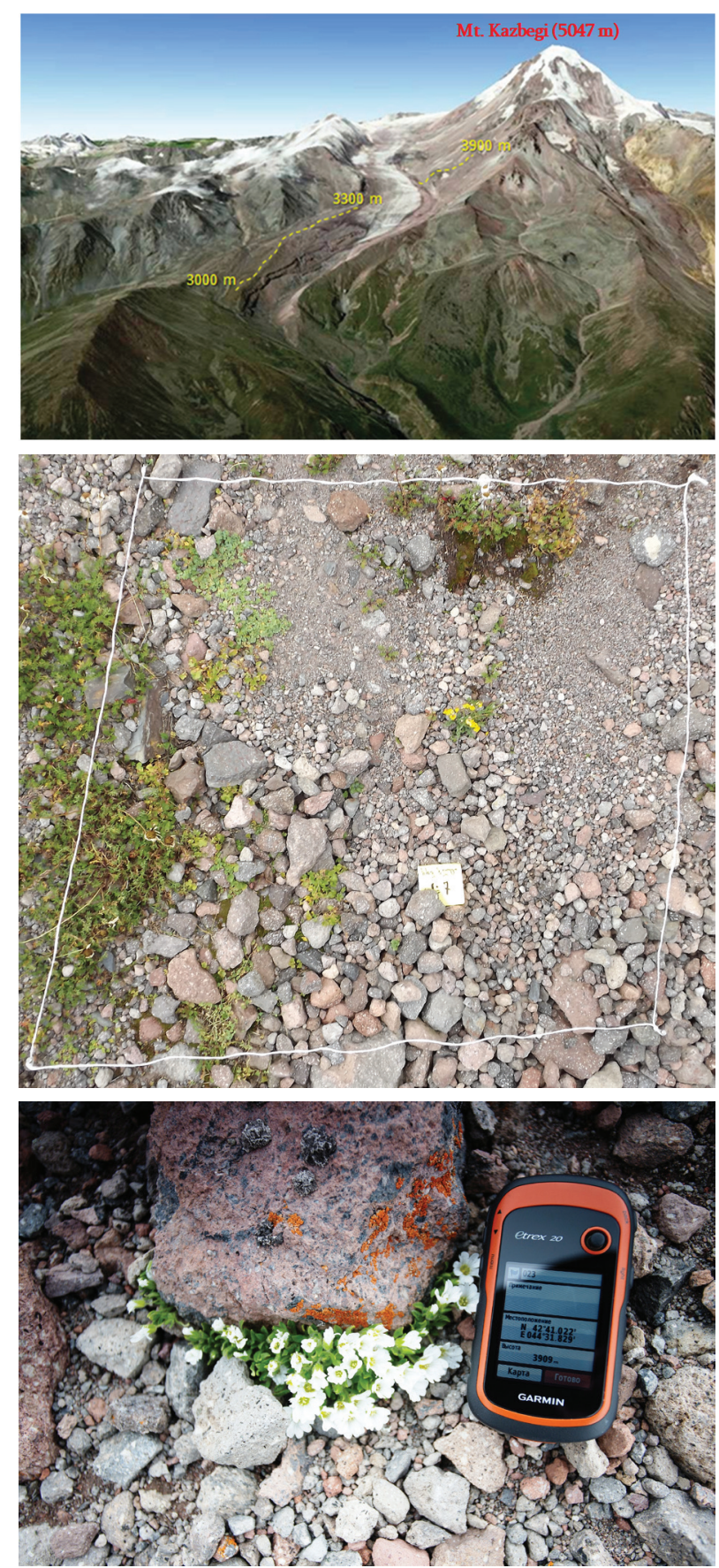

Fig. 3. Upper panel: Approximate location of the study transect on the slopes of Mt. Kazbegi (the Central Great Caucasus); Middle panel: A sampling plot ( $1 \mathrm{~m}^{2}$ square $)$; Lower panel: mini logger and GPS device

a flame atomic absorption spectrometer (Zeenit 700p, Analytik Jena, Germany).

Ukrainian Botanical Journal, 2021, 78(3) 
The soil temperature of each plot was measured with a temperature logger (DS1922L-F5\#-Termochron iBotton, USA). The loggers were buried in the centre of one of the plots (selected at random at each of 14 sampling sites along the transect) at the soil depth of $10 \mathrm{~cm}$ (Scherrer, Körner, 2010). The soil temperature was measured automatically every 2 minutes, mean values were calculated and recorded every 2 hours. The recording started on 19.08.2019, and was performed each day from 12:00:01 am to 10:00:01 pm, over full one year. In total the loggers produced 42,000 temperature data, from where we calculated the mean maximum temperature for each day and then the mean maximum annual temperature, for each sampling site.

\section{Data analyses}

We constructed a community matrix which included: elevation ( $m$ a.s.l.), slope angle (inclination), slope aspect, soil $\mathrm{pH}$, plant available N, P, K, AMMT, SOM (\%), vegetation projective cover (\%) and plant community data (plant species and their frequency of occurrence). In our previous work we found that vegetation cover was reducing exponentially with increasing elevation (Kikvidze et al., 2020), and for statistical analyses we logtransformed cover data assuming a linear dependence of log-transformed cover on elevation. We used correlation analysis and multivariate ordination methods such as nonMetric Multidimensional Scaling (NMDS; Legendre, Gallagher, 2001), Principal Component Analysis (PCA; Mason, Gunst, 1985), and Canonical Correspondence Analysis (CCA; Palmer, 1993). In multivariate ordination only species with high frequency of occurrence $(>10)$ were analysed. There were 27 such species out of 63 species recorded in total (Table 2).

\section{Results}

The variables that we measured to characterize soil conditions showed strong dependence on elevation: while soil $\mathrm{pH}$ increased and reached its maximum values, plant available nutrients, soil organic matter and plant cover went down to 0 or nearly so levels, soil maximum temperatures also decreased to ca. $60 \%$ of their maximum value (Fig. 4). This similarity in behaviors suggest that these variables are notably collinear. Indeed, correlation among them appeared to be generally high and varied from $r=-0.68$ (slope inclination and plant available $\mathrm{K}$ ) to $r=-0.97$ (soil $\mathrm{pH}$ and soil organic matter; Table 3). Certainly, terrain variables (elevation, inclination and
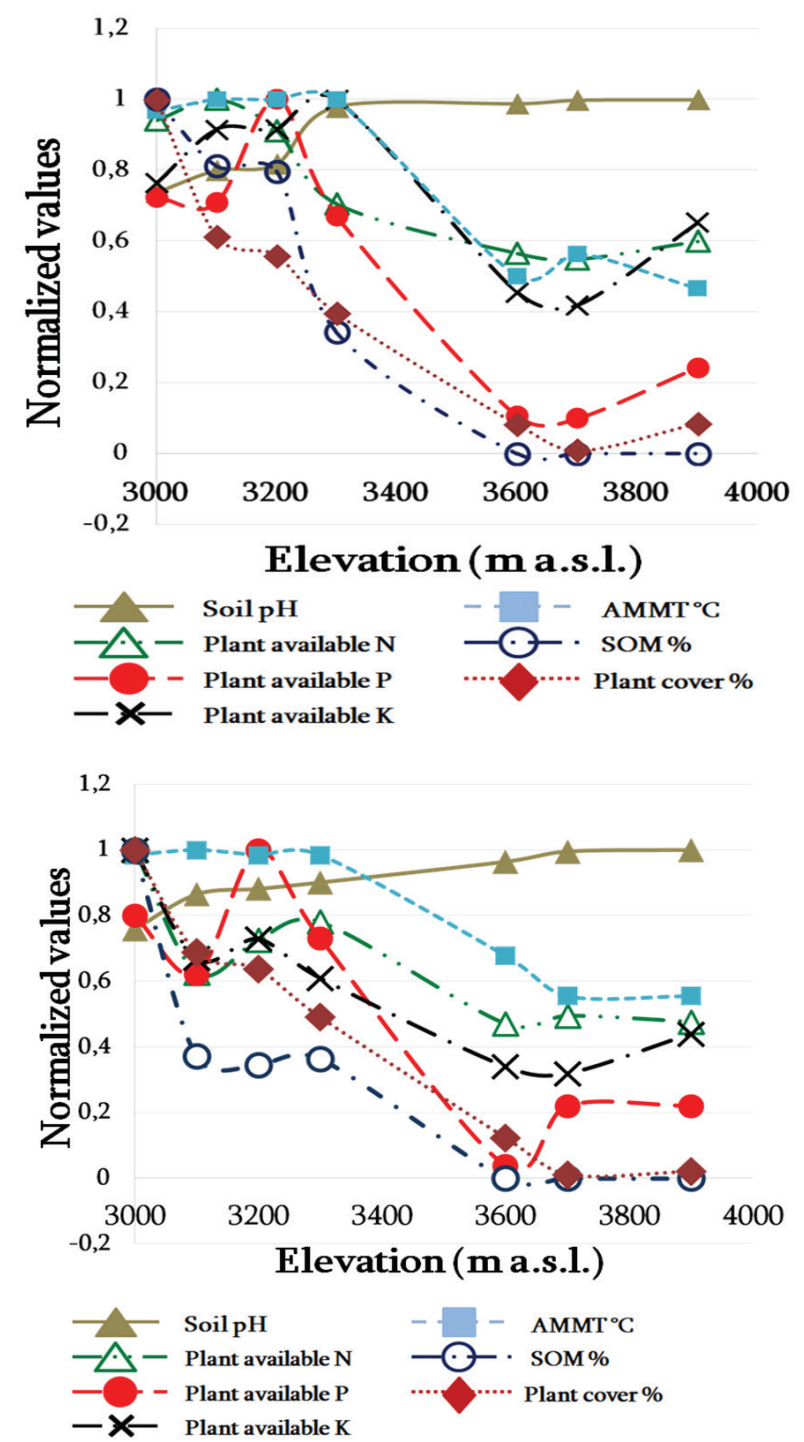

Fig. 4. Spatial distribution of soil characteristics - soil pH, Plant available NPK, Annual Mean Maximum Temperature (AMMT, $\mathrm{C}^{\circ}$ ), Soil Organic Matter (SOM, \%) and Plant cover (\%) along elevation gradient in alpine desert of Mt. Kazbegi.

Upper panel: North aspect; Lower panel: South aspect. Data were normalized by dividing their value by the maximum value of a given variable found on the gradient, the maximum value was expressed as 1

aspect) affect soil variables and contribute importantly to their change along the gradient. This dependence could be analysed using non-Metric Dimensional Scaling in which elevation, inclination and aspect were used as environmental (independent) variables and the rest were treated as dependent variables (Fig. 5). The ordination was satisfactory with the value of stress on data points as low as 0.0215 . nMDS yielded a very high R-squared value for the first coordinate (nMDS $1 ; \mathrm{R}^{2}=0.98$ ), and 
Table 2. Environmental parameters and frequencies of species in the dataset. The species with high of occurrence $(>10)$ are separated by a bold font ( 27 species)

\begin{tabular}{|c|c|c|c|c|c|c|c|c|c|c|c|c|c|c|}
\hline Elevation/Aspect & $\begin{array}{l}\text { Z } \\
\text { o } \\
\text { d }\end{array}$ & $\begin{array}{l}z \\
\stackrel{z}{\sigma} \\
\stackrel{m}{m}\end{array}$ & $\begin{array}{l}\text { Ž } \\
\text { ¿ } \\
\text { లె }\end{array}$ & 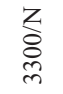 & 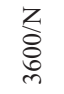 & 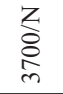 & $\begin{array}{l}\text { ž } \\
\text { ఏ } \\
\text { ळे }\end{array}$ & $\begin{array}{l}n \\
\text { ¿̊ } \\
\text { ò }\end{array}$ & $\frac{n}{\delta}$ & $\begin{array}{l}\text { na } \\
\stackrel{\text { Oे }}{\text { nd }}\end{array}$ & 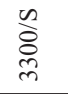 & $\begin{array}{l}\text { na } \\
\text { \& } \\
\text { d } \\
\text { r }\end{array}$ & $\underset{\infty}{\stackrel{n}{\infty}}$ & $\begin{array}{l}\infty \\
\text { ò } \\
\text { ळे }\end{array}$ \\
\hline Vegetation projective cover $\%$ & 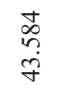 & 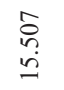 & $\begin{array}{l}\stackrel{\infty}{\infty} \\
\stackrel{\infty}{=} \\
=\end{array}$ & $\begin{array}{l}n \\
\tilde{0} \\
\infty \\
i\end{array}$ & $\frac{a}{\tilde{~}}$ & 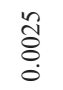 & 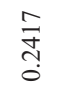 & $\begin{array}{l}\tilde{n} \\
\stackrel{n}{n}\end{array}$ & $\begin{array}{l}\stackrel{ \pm}{\Xi} \\
\stackrel{+}{ \pm}\end{array}$ & \begin{tabular}{l}
$\mathscr{2}$ \\
\multirow{2}{=}{} \\
$=$
\end{tabular} & 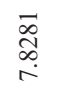 & 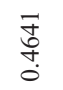 & $\stackrel{\circ}{\circ}$ & 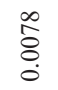 \\
\hline Slope inclination, degrees ${ }^{\circ}$ & 17 & 19 & 22 & 30 & 35 & 32 & 30 & 23 & 22 & 25 & 33 & 32 & 33 & 35 \\
\hline Plant available N (average) & $\begin{array}{l}\stackrel{8}{\circ} \\
\text { ते }\end{array}$ & $\begin{array}{l}\infty \\
\infty \\
\stackrel{\infty}{\infty}\end{array}$ & $\begin{array}{l}\stackrel{0}{0} \\
\stackrel{\infty}{\sim}\end{array}$ & $\stackrel{尺}{\stackrel{\sim}{i}}$ & $\stackrel{\stackrel{\vartheta}{\sim}}{\underset{\sim}{\sim}}$ & مِ & $\begin{array}{l}0 \\
\stackrel{+}{0} \\
\infty \\
-\end{array}$ & $\begin{array}{l}\stackrel{0}{1} \\
\stackrel{0}{0}\end{array}$ & $\begin{array}{l}\stackrel{8}{ } \\
\text { ì }\end{array}$ & 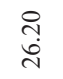 & 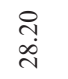 & $\begin{array}{l}\infty \\
\stackrel{0}{0}\end{array}$ & $\begin{array}{l}0 \\
\infty \\
\end{array}$ & $\vec{N}$ \\
\hline Plant available P (average) & $\hat{a}$ & $n$ & $\stackrel{+}{\sim}$ & $\stackrel{\circ}{\circ}$ & $\stackrel{\mathscr{P}}{\sim}$ & $\stackrel{\vec{m}}{-}$ & $\stackrel{\sim}{\sim}$ & $\stackrel{ナ}{\circ}$ & $\vec{\infty}$ & $\stackrel{0}{\ddot{2}}$ & $a ?$ & $\stackrel{\leftrightarrow}{\circ}$ & $\begin{array}{l}\stackrel{\circ}{\infty} \\
\stackrel{0}{i}\end{array}$ & $\begin{array}{l}\stackrel{2}{\infty} \\
\stackrel{i}{i}\end{array}$ \\
\hline AMMT, $\mathrm{C}^{\circ}$ & 31.1 & 32.1 & 32.1 & 32.1 & 16.1 & 18.1 & 15.0 & 32.1 & 32.6 & 32.1 & 32.1 & 22.1 & 18.1 & 18.1 \\
\hline SOM (\%, average) & 2.45 & 1.99 & 1.95 & 0.84 & 0 & 0 & 0 & 2.58 & 0.96 & 0.89 & 0.94 & 0 & 0 & 0 \\
\hline Alchemilla caucasica & 12 & . & . & . & . & . & . & 6 & 1 & $\cdot$ & . & . & . & $\cdot$ \\
\hline Alchemilla chlorosericea & 1 & . & . & . & & . & . & 4 & . & . & . & & . & . \\
\hline Alchemilla sericea & 6 & . & . & . & . & . & . & 2 & . & . & . & . & . & . \\
\hline Alopecurus glacialis & 84 & 21 & 8 & 18 & · & · & · & 62 & 12 & 4 & 5 & $\cdot$ & · & · \\
\hline Alopecurus laguroides & . & . & . & . & . & . & . & . & . & . & 2 & . & . & . \\
\hline Campanula ciliata & 28 & 4 & . & . & . & . & . & . & 9 & . & . & . & . & . \\
\hline Campanula biebersteiniana & 4 & . & . & . & . & . & . & . & . & . & . & . & . & 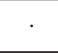 \\
\hline Carex dacica & 4 & . & . & . & . & . & . & . & . & . & . & . & . & . \\
\hline Carex tristis & 48 & . & . & . & . & . & . & 1 & 4 & . & . & . & . & $\cdot$ \\
\hline Cerastium kasbek & . & . & . & . & 20 & 16 & 13 & . & . & . & . & 60 & 33 & 22 \\
\hline Cerastium undulatifolium & . & . & . & . & . & . & . & . & 2 & . & . & . & . & . \\
\hline Cirsium obvallatum & 1 & . & . & . & . & . & . & . & . & . & . & . & . & . \\
\hline Colpodium versicolor & 17 & 8 & 3 & . & . & . & . & . & 13 & 10 & . & . & . & $\cdot$ \\
\hline Daphne glomerata & 1 & . & . & . & . & . & . & . & . & . & . & . & . & . \\
\hline Delphinium caucasicum & 3 & · & 1 & . & . & . & $\cdot$ & 25 & 10 & . & . & . & . & $\cdot$ \\
\hline Draba siliquosa & . & 9 & 14 & . & . & . & . & . & 4 & 11 & . & . & . & $\cdot$ \\
\hline Draba supranivalis & . & . & . & 2 & . & . & . & . & . & . & 7 & . & . & $\cdot$ \\
\hline Dryopteris oreades & 1 & . & . & . & . & . & . & . & . & . & . & . & . & . \\
\hline Erigeron uniflorus & 15 & 21 & . & . & . & . & . & 16 & 10 & 29 & . & . & . & $\cdot$ \\
\hline
\end{tabular}




\begin{tabular}{|c|c|c|c|c|c|c|c|c|c|c|c|c|c|c|}
\hline Veronica telephiifolia & 6 & 20 & 21 & 28 & . & . & . & . & 1 & . & 25 & . & . & . \\
\hline Hieracium sp. & 3 & . & . & . & . & . & $\cdot$ & . & . & . & . & . & . & · \\
\hline Jurinea filicifolia & 1 & . & . & . & . & . & . & . & 3 & . & . & . & . & . \\
\hline Luzula spicata & 1 & . & . & . & . & . & . & . & . & . & . & . & . & . \\
\hline $\begin{array}{l}\text { Minuartia circassica (now accepted as } \\
\text { Cherleria circassica) }\end{array}$ & 1 & . & 1 & . & . & . & . & . & 1 & . & . & . & . & . \\
\hline Minuartia oreina & 13 & 3 & . & $\cdot$ & . & . & . & 1 & 2 & . & . & . & . & . \\
\hline Myosotis alpestris & 1 & . & . & . & . & . & . & 3 & . & . & . & . & . & . \\
\hline Poa caucasica & . & . & . & . & . & . & . & . & . & 7 & 1 & . & . & . \\
\hline Potentilla gelida & . & . & . & $\cdot$ & . & . & . & 5 & . & . & . & . & . & · \\
\hline Saxifraga flagellaris & 19 & 11 & 1 & . & . & . & . & 11 & 16 & 4 & . & . & . & . \\
\hline Saxifraga juniperifolia & 12 & . & . & . & . & . & . & . & . & . & . & . & . & . \\
\hline Saxifraga kartilaginea & . & . & . & . & . & . & . & 1 & . & . & . & . & . & . \\
\hline Saxifraga moschata & 41 & 5 & 3 & . & . & 2 & . & 40 & 15 & 34 & 2 & . & 3 & . \\
\hline Saxifraga scleropoda & 1 & . & . & . & . & . & . & . & . & . & . & . & . & . \\
\hline Senecio taraxacifolius & 1 & 1 & . & . & . & . & . & . & 4 & . & . & . & . & . \\
\hline Sibbaldia parviflora & 53 & 1 & . & . & . & . & . & 9 & . & . & . & . & . & . \\
\hline Silene pygmaea & 4 & . & & . & . & . & . & . & . & . & . & . & . & . \\
\hline Taraxacum porphyranthum & 9 & 3 & . & . & . & . & . & 7 & 1 & 2 & . & . & . & . \\
\hline Taraxacum stevenii & 2 & . & . & . & . & . & . & . & . & . & . & . & . & . \\
\hline Tephroseris karjaginii & . & . & 1 & . & . & . & . & 11 & 1 & 4 & . & . & . & . \\
\hline Trifolium polyphyllum & 4 & . & . & . & . & · & . & . & . & . & . & . & . & · \\
\hline Tripleurospermum caucasicum & . & 3 & . & $\cdot$ & . & . & . & . & . & . & . & . & . & . \\
\hline Tripleurospermum subnivale & 45 & 110 & 55 & 7 & . & . & . & 11 & 44 & 39 & 8 & . & . & . \\
\hline Trisetum spicatum & 1 & . & . & . & . & . & . & . & 2 & . & . & . & . & \\
\hline Veronica schistosa & 4 & . & . & . & . & . & . & . & . & . & . & . & . & . \\
\hline Veronica gentianoides & 1 & . & . & . & . & . & . & . & . & . & . & . & . & . \\
\hline Viola minuta & 2 & . & . & . & . & . & . & . & . & . & . & . & . & · \\
\hline Ziziphora pushkinii & 1 & . & . & . & . & . & . & 9 & . & . & . & . & . & . \\
\hline Ziziphora subnivalis & 6 & . & . & . & . & . & . & . & 1 & . & . & . & . & . \\
\hline
\end{tabular}


Table 3. Correlation matrix among the main variables interplaying along elevation transect in the subnival-nival belt (3000$3900 \mathrm{~m}$ a.s.l.) in alpine desert of Mt. Kazbegi (Variables: elevation, $\mathrm{m}$ a.s.1.; Inclination, degrees ${ }^{\circ}$; Soil $\mathrm{pH}$ in $\mathrm{pH}$ units; $\mathrm{Plant}$ available nutrient $[\mathrm{N}, \mathrm{P}, \mathrm{K}], \%$; AMMT = Annual Mean Maximum Temperature, $\mathrm{C}^{\circ} ; \mathrm{SOM}=$ Soil Organic Matter, \%; Plant cover $=$ vegetation projective cover as log-transformed \%)

\begin{tabular}{|l|c|c|c|c|c|c|c|c|c|}
\hline \multicolumn{1}{|c|}{ Variables } & Elevation & Inclination & Soil pH & Available N & Available P & Available K & AMMT & SOM & Plant Cover \\
\hline Elevation & $\mathrm{X}$ & $<0.0001$ & $<0.0001$ & $<0.0001$ & $<0.0001$ & $<0.0001$ & $<0.0001$ & $<0.0001$ & $<0.0001$ \\
\hline Inclination & 0.82 & $\mathrm{X}$ & $<0.0001$ & $<0.0001$ & $<0.0001$ & 0.01 & $<0.0001$ & $<0.0001$ & $<0.0001$ \\
\hline Soil pH & 0.89 & 0.9 & $\mathrm{X}$ & $<0.0001$ & $<0.0001$ & $<0.0001$ & $<0.0001$ & $<0.0001$ & $<0.0001$ \\
\hline Available N & -0.86 & -0.75 & -0.91 & $\mathrm{X}$ & $<0.0001$ & $<0.0001$ & $<0.0001$ & $<0.0001$ & $<0.0001$ \\
\hline Available P & -0.83 & -0.72 & -0.76 & 0.84 & $\mathrm{X}$ & $<0.0001$ & $<0.0001$ & $<0.0001$ & $<0.0001$ \\
\hline Available K & -0.78 & -0.68 & -0.71 & 0.84 & 0.86 & $\mathrm{X}$ & $<0.0001$ & $<0.0001$ & $<0.0001$ \\
\hline AMMT & -0.93 & -0.72 & -0.77 & 0.81 & 0.9 & 0.81 & $\mathrm{X}$ & $<0.0001$ & $<0.0001$ \\
\hline SOM & -0.89 & -0.87 & -0.97 & 0.94 & 0.8 & 0.82 & 0.8 & $\mathrm{X}$ & $<0.0001$ \\
\hline Plant cover & -0.88 & -0.76 & -0.81 & 0.81 & 0.82 & 0.83 & 0.87 & 0.81 & $\mathrm{X}$ \\
\hline
\end{tabular}

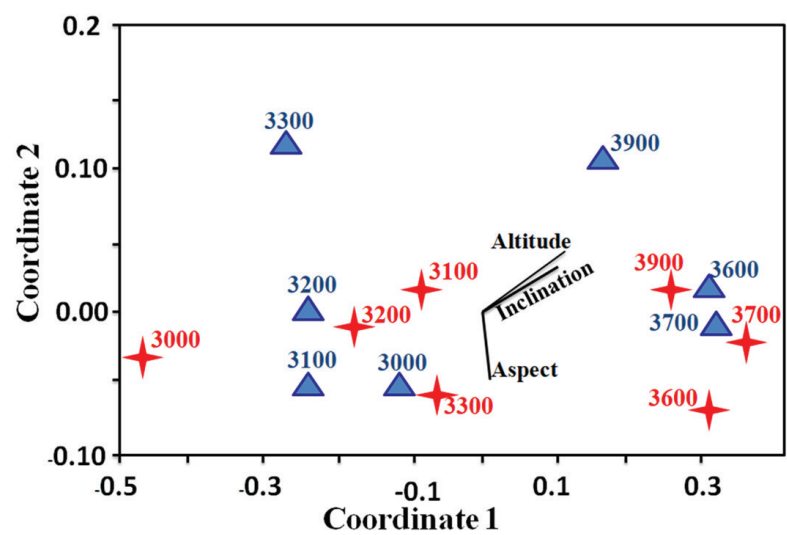

Fig. 5. Non-metric multidimensional scaling (NMDS) of environmental variables along the transect on two slope aspects. Elevation, Inclination and Slope aspect are used as independent variables; soil $\mathrm{pH}$, Plant available nutrients (NPK), Annual Mean Maximum Temperature (AMMT, $\mathrm{C}^{\circ}$ ), Soil Organic Matter (SOM, \%) and Plant cover (\%, log-transformed) as dependent variables

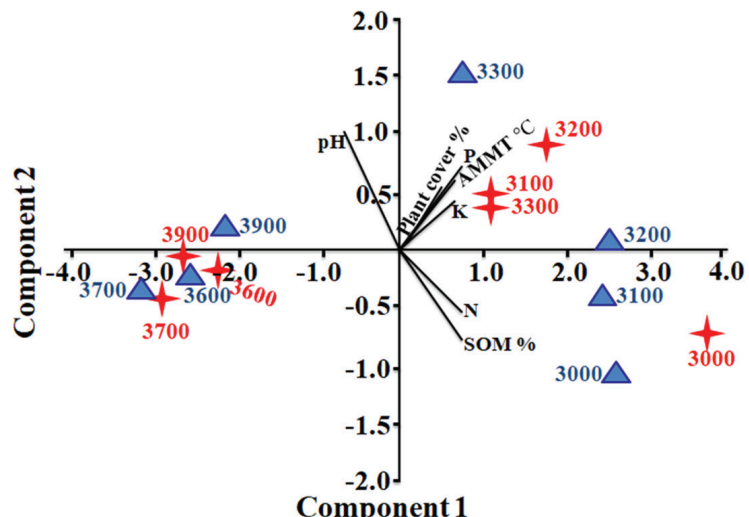

Fig. 6. PCA biplot of environmental variables along the transect. Soil pH, Plant available nutrients (NPK), Annual Mean Maximum Temperature (AMMT, ${ }^{\circ} \mathrm{C}$ ), Soil Organic Matter (SOM, \%) and Plant cover (\%, log-transformed) were analyzed. The first two components of PCA explain over $92 \%$ of the variance in the data sampling sites arranged along this axis from low to high suggesting that nMDS 1 primarily represents elevation. The second coordinate (nMDS 2) produced a 0 level of R-squared, probably it represents a rather idiosyncratic variability mostly observed on the slopes of different aspect (Jolokhava et al., 2020).

As an independent test, we used the PCA ordination to look at soil variables. The results were very similar to those from the nMDS (Fig. 6). Like the nMDS, the PCA produced a satisfactory ordination, in which the first two components explained up to $92 \%$ of variation in data. The PCA graph helps visualize the collinearity of soil variables and corroborates with the results of correlation analyses (Table 2). We observed also a close correlation among the axes of the two ordination methods: the correlation was very tight between nMDS 1 and PCA $1(r=-0.96, p<0.0001)$ and quite strong between nMDS 2 and PCA $2(r=0.61, p=0.02)$. These ordination axes showed distinctive correlations with the terrain and soil variables (Table 4). nMDS 1 and PCA1 were most strongly correlated with plant available $\mathrm{K}$ and $\mathrm{N}$, respectively, whilst nMDS 2 and PCA 2 correlated most strongly with soil $\mathrm{pH}$.

Ordination axes can be used as composite gradients that combine several, most strongly correlated variables (Palmer, 1993). Besides, the observed relationships helped select environmental variables that could be used for the analysis of plant species distribution: these variables can be chosen on the basis of the fact that they correlate most strongly with ordination axes assuming that the selected variables represent the largest part of variability along the gradient. For the analysis of plant species distributions we used the CCA ordination. All in all, four pairs of environmental variables were compared in our CCA computations: (1) nMDS 1 and nMDS 2, (2) PCA 1 and PCA 2, (3) Plant available $\mathrm{N}$ and soil pH, and (4) Plant available $\mathrm{K}$ and soil $\mathrm{pH}$. The $\mathrm{CCA}$ produced 
Table 5. Results of the CCA ordination with different environmental variables along Eleveation transect in the subnival-nival belt (3000-3900 $\mathrm{m}$ a.s.l.) in alpine desert of Mt. Kazbegi using composite and best-fit environmental variables (Environmental variables: Plant available nutrient $[\mathrm{N}, \mathrm{K}], \%$; Soil $\mathrm{pH}$ in $\mathrm{pH}$ units). Bold font indicates maximum Eigenvalue for a given composite variable system and minimum $p$-value

\begin{tabular}{|l|c|c|c|c|}
\hline \multicolumn{1}{|c|}{ Method } & Axis & Eigenvalue & $\%$ & P-Value \\
\hline nMDS 1 & CCA1 & 0.313 & 65.5 & 0.017 \\
\hline nMDS 2 & CCA2 & 0.165 & 34.5 & 0.087 \\
\hline PCA1 & CCA1 & 0.318 & 84.56 & 0.011 \\
\hline PCA2 & CCA2 & 0.058 & 15.44 & 0.808 \\
\hline Plant available N & CCA1 & 0.427 & 99.99 & 0.002 \\
\hline Soil pH & CCA2 & 0 & 0 & 0.262 \\
\hline Plant available K & CCA1 & $\mathbf{0 . 5 2 0}$ & 99.97 & $\mathbf{0 . 0 0 1}$ \\
\hline Soil pH & CCA2 & 0 & 0.0259 & 0.246 \\
\hline
\end{tabular}

satisfactory ordinations in all of these cases (Table 5). However, the highest Eigenvalues and lowest $p$-vales were obtained for plant available $\mathrm{K}$ and soil $\mathrm{pH}$ (Fig. 7). These two variables, therefore, appeared to be the best predictors of plant species distribution along elevation gradient in our data set.

\section{Discussion}

Our main finding is that, on the gradients of regional and local scale, replacing abiotic factors with soil properties can improve the models that describe species distribution along the gradient. High mountain environments are notable for the presence of short growing seasons and low temperatures of air and soil (Mooney, Billings, 1965; Peterson, Billings, 1982; Sakai, Larcher, 1987), which determine morphological and physiological adaptations of alpine plant species (Bliss, 1971; Billings, 1974). The importance of soils to the effects of environmental variation on different plant communities have been the subject of many ecological studies in recent years (Lyon, Gross, 2005; Bowles et al., 2014). At biogeographical scale it was found that soil and plant community types match well to climatic variables, which proved to be good predictors of species distributions along large gradients (Manley, 1961; Motzkin et al., 1999). The consequent models of species distribution at this scale usually omit the role of soils considering them to be the part of response to variation in abiotic conditions. However, this approach might not be as good on regional and local gradients, and a refinement that envisages a mediating role of soils between the climate and vegetation might improve the accuracy of these models.

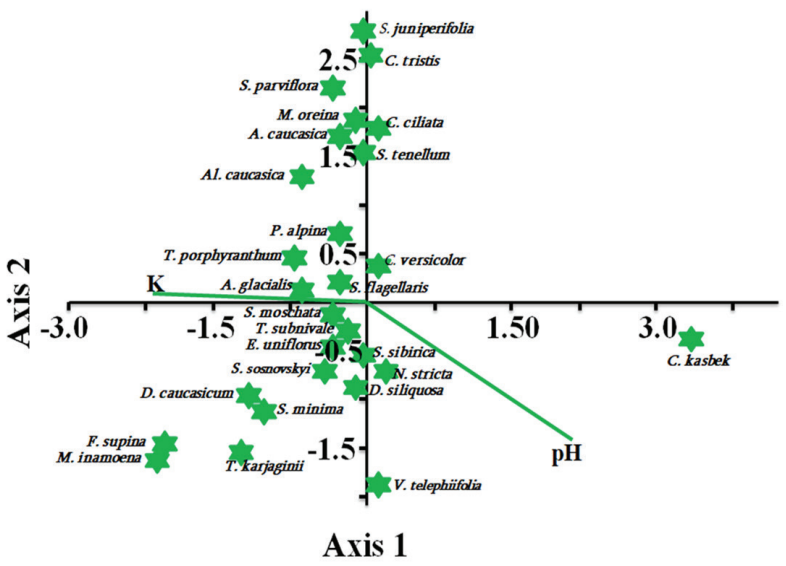

Fig. 7. CCA ordination of plant species distribution along elevation gradient in alpine desert of Mt. Kazbegi (3000-3900 m a.s.l.). Plant available $\mathrm{K}$ and Soil $\mathrm{pH}$ are used as environmental variables (Species full names are given in Table 2)

Soils of mountain environments are highly variable over short spatial scales (Baruck et al., 2016), partly due to the heterogeneity of geological substrates and highly dynamic geomorphic processes (Birkland et al., 2003; Chase et al., 2012). The Caucasus and, particularly, the Great Caucasus show high soil diversity owing to complex bioclimatic, bedrock and geomorphologic conditions (Urushadze, 1989). An important step in the inclusion of soils into distribution models can be the recognising that soils play and intermediary role by connecting climatic and other abiotic variables to vegetation (Jenny, 1994). This role can be effectively revealed on an elevation gradient (Chapin, Körner, 1994; Körner, 2003; Rezaei, Gilkes, 2005). In particular, the low part of our transect represents the alpine-nival ecotone with high representation of alpine meadow plant species ("alpine carpets" sensu Nakhutsrishvili, 2012), which are replaced by "typical nival" species higher up (Nakhutsrishvili, Gamtsemlidze, 1984; Nakhutsrishvili, Gagnidze, 1999). The articulation of soil as a mediator between the abiotic environment and the plants allowed us to see how soil properties changed along a variable abiotic environment and how these properties correlated with species distributions. As mentioned above, this correlation appeared to be stronger than when abiotic variables were replaced by soil properties.

The interplay of biotic and abiotic processes can actually determine the relative abundances of species (Wisz et al., 2013), and soil is precisely the component that in a way integrates and modifies the variation in abiotic variables. For example, two sites can receive similar sun energy at two different elevations depending 
whether they are exposed to north or south. Likewise, a site on a steeper slope can have different water relations than other sites on milder slope at the same elevation and aspect (Geiger et al., 1995; Barry, 2008). In other words, sites with similar soil properties including water and temperature relations can correspond to slightly different elevations, which is the main driver of variation in abiotic environment in our system, aspects and inclinations, and this adds certain statistical noise to the models that include abiotic variables. Models based only on soil properties are free from such influence.

The vegetation of subnival and nival belts is mostly represented by slow-growing and long-living perennials, with only a few annual species (Nagy et al., 2003) that could be used as bioindicators (Grabherr et al., 1995; Körner, 2003). This vegetation with high endemic rates (Nakhutsrishvili et al., 2005, 2006) currently attracts special interest owing to its vulnerability to climate changes (Gigauri et al., 2016) and we believe that our results have clear implications for analysing and monitoring species distributions in these bioma. How important is the amendment that bases gradient models solely on soil properties, and will it hold in other systems? An answer obviously requires more research, and we strongly believe that improving the gradient models for the monitoring and explaining of species distribution at regional and local scale can be a priority for future research.

\section{Conclusions}

We conclude that the models that account for the role of soils as a mediator between the abiotic environment and vegetation can more accurately describe plant species distribution at local and regional scales: a potentially important amendment with implications for the monitoring of climate change effects on vegetation, at least in high mountain systems.

\section{Acknowledgements}

We are grateful to Ilia State University for financial and technical support of the project. At the same time this work would not have been possible without the support of many people. First of all, we would like to express our gratitude to Prof. Dr. George Nakhutsrishvili for providing his valuable guidance in this project. We thank Dr. Zezva Asanidze, researcher Arsen Bakhia, and students of Ilia State University for their help during fieldwork.

\section{References}

Abdaladze O., Nakhutsrishvili G., Batsatsashvili K., Gigauri Kh., Jolokhava T., Mikeladze G. 2015. Sensitive alpine plant communities to the global environmental changes (Kazbegi Region, the Central Great Caucasus). American Journal of Environmental Protection, 4: 93100. https://doi.org/10.11648/j.ajep.s.2015040301.25

Barry R.G. 2008. Mountain Weather and Climate, $3^{\text {rd }}$ ed. Cambridge: Cambridge University Press, 506 pp.

Baruck J., Nestroy O., Sartori G., Baize D., Traidl R., Vrščaj B., Bräm E., Gruber F.E., Heinrich K., Geitner C. 2016. Soil classification and mapping in the Alps: The current state and future challenges. Geoderma, 264: 312 331. https://doi.org/10.1016/j.geoderma.2015.08.005

Bezemer T., Lawson C.S., Hedlund K., Edwards A.R., Brook A.J., Igual J.M., Mortimer S.R., van Derputten W.H. 2006. Plant species and functional group effects on abiotic and microbial soil properties and plant-soil feedback responses in two grasslands. Journal of Ecology, 94: 893904. https://doi.org/10.1111/j.1365-2745.2006.01158.x

Billings W.D. 1974: Adaptations and origins of alpine plants. Arctic and Alpine Research, 6: 129-142. https://doi.org/10 $.1080 / 00040851.1974 .12003769$

Birkeland P.W., Shroba R.R., Burns S.F., Price A.B., Tonkin P.J. 2003. Integrating soils and geomorphology in mountains - an example from the Front Range of Colorado. Geomorphology, 55(1-4): 329-344. https://doi. org/10.1016/S0169-555X(03)00148-X

Bliss L.C. 1971. Arctic and alpine plant life cycles. Annual Review of Ecology and Systematics, 2: 405-438. https:// doi.org/10.1146/annurev.es.02.110171.002201

Bowles T.M., Acosta-Martínez V., Calderón F., Jackson L.E. 2014. Soil enzyme activities, microbial communities, and carbon and nitrogen availability in organic agroecosystems across an intensively-managed agricultural landscape. Soil Biology and Biochemistry, 68: 252-262. https://doi. org/10.1016/j.soilbio.2013.10.004

Brevik E.C. 2013. The potential impact of climate change on soil properties and processes and corresponding influence on food security. Agriculture, 3(3): 398-417. https://doi. org/10.3390/agriculture3030398

Budzhak V.V., Didukh Ya.P. 2020. Ukrainian Botanical Journal, 77(6): 434-453. [Буджак В.В., Дідух Я.П. 2020. Синфітоіндикаційна оцінка оселищ рослин Червоної книги України та ризиків їхніх втрат під впливом кліматогенних змін. Украӥнський ботанічний журнал, 77(6): 434-453]. https://doi.org/10.15407/ ukrbotj77.06.434

Buri A., Grand S., Yashiro E., Adatte T., Spangenberg J.E., Pinto-Figueroa E., Verrecchia E., Guisan A. 2020. What are the most crucial soil variables for predicting the distribution of mountain plant species? A comprehensive study in the Swiss Alps. Journal of Biogeography, 47(5): 1143-1153. https://doi.org/10.1111/jbi.13803

Chapin F.S., Shaver G.R., Giblin A.E., Nadelhoffer K.J., Laundre J.A. 1995. Responses of Arctic tundra to 
experimental and observed changes in climate. Ecology, 76: 694-71. https://doi.org/10.2307/1939337

Chapin III F.S., Körner C. 1994. Arctic and alpine biodiversity: patterns, causes and ecosystem consequences. Trends in Ecology \& Evolution, 9(2): 45-47. https://doi. org/10.1016/0169-5347(94)90266-6

Chase M.N., Johnson E.A. Johnson., Martin Y.E. 2012. The influence of geomorphic processes on plant distribution and abundance as reflected in plant tolerance curves. Ecological Monographs, 82: 429-447. https://doi. org/10.1890/11-2145.1

Dahlgren R.A. 2006. Biogeochemical processes in soils and ecosystems: from landscape to molecular scale. Journal of Geochemical Exploration, 88: 186-189. https://doi. org/10.1016/j.gexplo.2005.08.035

Devictor V., Mouillot D., Meynard C., Jiguet F., Thuiller W., Mouquet N. 2010. Spatial mismatch and congruence between taxonomic, phylogenetic and functional diversity: the need for integrative conservation strategies in a changing world. Ecology Letters, 13(8): 1030-1040. https://doi.org/10.1111/j.1461-0248.2010.01493.x

Donhauser J., Frey B. 2018. Alpine soil microbial ecology in a changing world. FEMS Microbiology Ecology, 94(9), fiy099. https://doi.org/10.1093/femsec/fiy099

Eisenhauer N., Dobies T., Cesarz S., Hobbie S.E., Meyer R.J., Worm K., Reich P.B. 2013. Plant diversity effects on soil food webs are stronger than those of elevated $\mathrm{CO}^{2}$ and $\mathrm{N}$ deposition in a long-term grassland experiment. Proceedings of the National Academy of Sciences, 110(17): 6889-6894. https://doi.org/10.1073/pnas.1217382110

Gavlak R., Horneck D., Miller R.O., Kotuby-Amacher J. 2003. Soil, plant and water reference methods for the western region. Fort Collins, CO, USA: WCC-103 Publication, $206 \mathrm{pp}$.

Geiger R., Aron T.R.H., Todhunter P. 1995. The Climate Near the Ground. Vieweg+Teubner Verlag: Springer, 528 pp.

Gigauri K., Akhalkatsi M., Abdaladze O., Nakhutsrishvili G. 2016. Alpine plant distribution and thermic vegetation indicator on GLORIA summits in the Central Greater Caucasus. Pakistan Journal of Botany, 48(5): 1893-902.

Gleason H.A. 1926. The individualistic concept of the plant association. Bulletin of the Torrey Botanical Club, 53(1): 7-26. https://doi.org/10.2307/2479933

Grabherr G., Gottfried M., Gruber A., Pauli H. 1995. Patterns and current changes in alpine plant diversity. In: Arctic and alpine biodiversity: patterns, causes and ecosystem consequences. Eds F.S. Chapin, Ch. Korner. Berlin, Heidelberg: Springer, pp. 167-181.

Hooper D.U., Bignell D.E., Brown V.K., Brussard L., Dangerfield J.M., Wall D.H. Wardle D.A., Coleman D.C., Giller K.E., Lavelle P., Van Der Putten W.H. 2000. Interactions between Aboveground and Belowground Biodiversity in Terrestrial Ecosystems: Patterns, Mechanisms, and Feedbacks: We assess the evidence for correlation between aboveground and belowground diversity and conclude that a variety of mechanisms could lead to positive, negative, or no relationshipdepending on the strength and type of interactions among species. Bioscience, 50(12): 1049-1061. https://doi. org/10.1641/0006-3568(2000)050[1049:IBAABB]2.0. $\mathrm{CO} ; 2$

Huston M.A., Huston M.A. 1994. Biological diversity: the coexistence of species on changing landscapes. United Kingdom: Cambridge University Press, 615 pp.

Jackson M.L. 2005. Soil chemical analysis: Advanced course. Madison, Wisconsin: Madison Libraries Parallel Press, 929 pp.

Jenny H. 1994. Factors of soil formation: a system of quantitative pedology. New York: Dover Publication INC, $191 \mathrm{pp}$.

Jolokhava T., Abdaladze O., Gadilia S., Kikvidze Z. 2020. Variable soil $\mathrm{pH}$ can drive changes in slope aspect preference of plants in alpine desert of the Central Great Caucasus (Kazbegi district, Georgia). Acta Oecologica, 105: 103582. https://doi.org/10.1016/j.actao.2020.103582

Kardol P., Martijn Bezemer T., Van Der Putten W.H. 2006. Temporal variation in plant-soil feedback controls succession. Ecology Letters, 9(9): 1080-1088. https://doi. org/10.1111/j.1461-0248.2006.00953.x

Kent M. 2011. Vegetation description and data analysis: a practical approach. London: John Wiley \& Sons. 80 pp.

Kharadze A.L. 1965. On the Subnival Zone of the Greater Caucasus. Notulae Systematicae ac Geographicae Intituti Botanici Thbilisiensis, 25: 103-114. [Харадзе А.Л. 1965. О субнивальном поясе Большого Кавказа. Заметки по систематике и географии растений Института ботаники АН ГССР (Тбилиси), 25: 103-114].

Kikvidze Z. 1993. Plant species associations in alpine-subnival vegetation patches in the Central Caucasus. Journal of Vegetation Science, 4(3): 297-302. https://doi. org/10.2307/3235587

Kikvidze Z., Nakhutsrishvili G. 1998. Facilitation in subnival vegetation patches. Journal of Vegetation Science, 9(2): 261-264. https://doi.org/10.2307/3237125

Kikvidze Z., Jolokhava T., Bakhia A., Abdaladze O. 2020. Jumping the barrier: does a glacier tongue affect species distribution along the elevation gradient in the subnival and nival belts? A case study on Mt. Kazbegi, Georgia, Central Great Caucasus Mountains. Botanica Serbica, 44(2): 219229. https://doi.org/10.2298/BOTSERB2002219K

Körner Ch., Paulsen J. 2017. A geostatistical and bioclimatological comparison of the Central Great Caucasus and the central Alps. In: Plant diversity in the Central Great Caucasus: a quantitative assessment. Eds G. Nakhutsrishvili, O. Abdaladze, K. Batsatsashvili, Ch. Körner, E. Spehn. Switzerland, Cham: Springer, pp. 1-9.

Körner Ch. 2003. Alpine plant life: functional plant ecology of high mountain ecosystems; with 47 tables. Switzerland: Springer Science \& Business Media. 343 pp.

Körner C. 2007. The use of 'altitude' in ecological research. Trends in Ecology \& Evolution, 22(11): 569-574. https:// doi.org/10.1016/j.tree.2007.09.006 
Körner Ch. 2011. Coldest place on Earth with angiosperm plant life. Alpine Botany, 121: 11-22. https://doi. org/10.1007/s00035-011-0089-1

Körner Ch., Larcher W. 1988. Plant life in cold climates. In: Symposia of the Society for Experimental Biology, 42: 25-57.

Legendre P., Gallagher E.D. 2001. Ecologically meaningful transformations for ordination of species data. Oecologia, 129(2): 271-280. https://doi.org/10.1007/s004420100716

Lomolino M.V. 2001. Elevation gradients of speciesdensity: historical and prospective views. Global Ecology and Biogeography, 10: 3-13. https://doi. org/10.1046/j.1466-822x.2001.00229.x

Lyon J., Gross N.M. 2005. Patterns of plant diversity and plant-environmental relationships across three riparian corridors. Forest Ecology and Management, 204(2-3): 267-278. https://doi.org/10.1016/j.foreco.2004.09.019

Manley G. 1961. Effects of climate on vegetation. Nature, 192: 5. https://doi.org/10.1038/192005a0

Maruashvili L. 1971. Geomorphology of Georgia. Tbilisi: Metsniereba, 346 pp. [Маруашвили Л.И. 1971. Геоморфология Грузии. Тбилиси: Мецниереба, 346 с.]

Mason R.L., Gunst R.F. 1985. Outlier-induced collinearities. Technometrics, 27(4), 401-407. https://doi.org/10.1080/00 401706.1985.10488079

Michalet R., Gandoy C., Joud D., Pagès J.P., Choler P. 2002. Plant community composition and biomass on calcareous and siliceous substrates in the northern French Alps: comparative effects of soil chemistry and water status. Arctic, Antarctic, and Alpine Research, 34(1): 102-113. https://doi.org/10.1080/15230430.2002.12003474

Molau U., Nordenhall U., Eriksen B. 2005. Onset of flowering and climate variability in an alpine landscape: a 10-year study from Swedish Lapland. American Journal of Botany, 92: 422-431. https://doi.org/10.3732/ajb.92.3.422

Mooney H.A., Billings W.D. 1965. Effects of altitude on carbohydrate content of mountain plants. Ecology, 46: 750-751. https://doi.org/10.2307/1935021

Motzkin G., Wilson P., Foster D.R., Allen A. 1999. Vegetation patterns in heterogeneous landscapes: the importance of history and environment. Journal of Vegetation Science, 10: 903-920. https://doi.org/10.2307/3237315

Nagy L., Grabherr G., Körner C., Thompson D.B.A. 2003. Alpine biodiversity in space and time: a synthesis. Berlin: Springer, Berlin, Heidelberg, 453-464 pp.

Nakhutsrishvili G.S. 1998. The vegetation of the subnival belt of the Caucasus Mountains. Arctic and Alpine Research, 30(3): 222-226.

Nakhutsrishvili G. 2003. High-mountain vegetation of the Caucasus region. In: Alpine Biodiversity in Europe. Eds L. Nagy, G. Grabherr, Ch. Korner, D.B.A. Thompson. BerlinHeidelberg: Springer, pp. 93-103.

Nakhutsrishvili G. 2012. The vegetation of Georgia (South Caucasus). Berlin-Heidelberg: Springer, 235 pp.

Nakhutsrishvili G., Gamtsemlidze Z.G. 1984. Plant life in extreme environment of high mountains. Leningrad: Nauka, 123 pp. [Нахучришвили Г.Ш., Гамиемлидзе 3.Г.
1984. Жизнь растений в экстремальных условиях выссокогорий. Ленинград: Наука, 123 с.].

Nakhutsrishvili G., Gagnidze R. 1999. Die subnivale und nivale Hochgebirgsvegetation des Kaukasus. Phytocoenosis, 11: 173-183.

Nakhutsrishvili G., Abdaladze O. 2017a. Plant diversity of the Central Great Caucasus. In: Plant diversity in the Central Great Caucasus: a quantitative assessment. Eds G. Nakhutsrishvili, O. Abdaladze, K. Batsatsashvili, Ch. Körner, E. Spehn. Switzerland, Cham: Springer, pp. 17-132.

Nakhutsrishvili G., Abdaladze O. 2017b. Vegetation of the Central Great Caucasus along W-E and N-S transects. In: Plant diversity in the Central Great Caucasus: a quantitative assessment. Eds G. Nakhutsrishvili, O. Abdaladze, K. Batsatsashvili, Ch. Körner, E. Spehn. Switzerland, Cham: Springer, pp. 11-16.

Nakhutsrishvili G., Abdaladze O., Kikodze A. 2005. Khevi: Kazbegi Region. Tbilisi: Institute of Botany, 54 pp.

Nakhutsrishvili G., Abdaladze O., Akhalkatsi M. 2006. Biotope types of the treeline of the Central Greater Caucasus. In: Nature Conservation: Concepts and Practice. Eds D. Gafta, J. Akeroyd. Berlin-Heidelberg: Springer, pp. 211-225.

Norman R.J., Stucki J.W. 1981. The determination of nitrate and nitrite in soil extracts by ultraviolet spectrophotometry. Soil Science Society of America Journal, 45(2): 347-353. https://doi.org/10.2136/sssaj1981.0361599500450002002 $\underline{4 \mathrm{x}}$

Olsen S.R. 1954. Estimation of available phosphorus in soils by extraction with sodium bicarbonate (No. 939). Washington: US Department of Agriculture. 1-19 pp.

Palmer M.W. 1993. Putting things in even better order: the advantages of canonical correspondence analysis. Ecology, 74(8): 2215-2230. https://doi.org/10.2307/1939575

Palmer M.W. 2004. Ordination methods - an overview. Oklahoma, Stillwater: Botany Department, Oklahoma State University, $27 \mathrm{pp}$.

Pansu M., Gautheyrou J. 2007. Handbook of soil analysis: mineralogical, organic and inorganic methods. New York: Springer, Berlin, Heidelberg, 993 pp.

Peterson K.M., Billings W.D. 1982: Growth of alpine plants under controlled drought. Arctic and Alpine Research, 14: 189-194.

Praeg N., Pauli H., Illmer P. 2019. Microbial diversity in bulk and rhizosphere soil of Ranunculus glacialis along a highalpine altitudinal gradient. Frontiers in microbiology, 10: 1429. https://doi.org/10.3389/fmicb.2019.01429

Rezaei S.A., Gilkes R.J. 2005. The effects of landscape attributes and plant community on soil chemical properties in rangelands. Geoderma, 125(1-2): 167-176. https://doi. or/10.1016/j.geoderma.2004.07.010

Sakai A., Larcher W. 1987. Frost survival of plants: Responses and adaptation to freezing stress. Berlin: Springer-Verlag, $321 \mathrm{pp}$.

Scherrer D., Körner C. 2010. Infra-red thermometry of alpine landscapes challenges climatic warming projections. 
Global Change Biology, 16(9): 2602-2613. https://doi. org/10.1111/j.1365-2486.2009.02122.x

Schulte E.E., Hopkins B.G. 1996. Estimation of soil organic matter by weight loss-on-ignition. Analysis and interpretation, 1(46): 21-31. https://doi.org/10.2136/ sssaspecpub46.c3

Solomon J.C., Shulkina T.V., Schatz G.E. 2014. Red list of the endemic plants of the Caucasus: Armenia, Azerbaijan, Georgia, Iran, Russia, and Turkey. St. Louis, Missouri: Missouri Botanical Garden Press, 451 pp.

Stuanes A.O., Ogner G., Opem M. 1984. Ammonium nitrate as extractant for soil exchangeable cations, exchangeable acidity and aluminum. Communications in Soil Science and Plant Analysis, 15(7): 773-778. https://doi. org/10.1080/00103628409367516

Talakhadze G.R., Urushadze T.F., Kirvalidze R.I. 1985. Pochvovedenie, 1: 156-159. [Талахадзе Г.Р., Урушадзе Т.Ф., Кирвалидзе Р.И. 1985. Роль Гедеванишвили Д.П. в становлении почвоведения в Грузии. Почвоведение, 1: 156-159].

Tale K.S., Ingole S. 2015. A review on role of physicochemical properties in soil quality. Chemical Science Review and Letters, 4(13): 57-66.
Tan K.H. 1995. Soil sampling, preparation, and analysis. New York: CRC Press, 84 pp.

IPNI. The International Plant Name Index. 2012-onward. Available at: http://www.ipni.org (Accessed 20 December 2017).

Tudela-Isanta M., Ladouceur E., Wijayasinghe M., Pritchard H.W., Mondoni A. 2018. The seed germination niche limits the distribution of some plant species in calcareous or siliceous alpine bedrocks. Alpine Botany, 128(1): 83-95. https://doi.org/10.1007/s00035-018-0199-0

Urushadze T. 1989. Mountain soils of the USSR. Moscow: Agropromizdat, 272 pp. [Урушадзе Т.Ф. 1989. Горныле почвы СССР. Москва: Агропромиздат, 272 с.].

Wisz M.S., Pottier J., Kissling W.D., Pellissier L., Lenoir J., Damgaard C.F., Dormann C.F., Forchhammer M.C., Grytnes J.A., Guisan A., Heikkinen R.K. 2013. The role of biotic interactions in shaping distributions and realised assemblages of species: implications for species distribution modelling. Biological Reviews, 88(1): 15-30. https://doi.org/10.1111/j.1469-185X.2012.00235.x

Recommended for publication by Ya.P. Didukh

\begin{abstract}
Джолохава Т., Абдаладзе О., Гігаурі Х., Кіквідзе 3. 2021. Градіснтний аналіз взаємодії грунт-рослини від альпійсько-нівального екотону до снігової лінії на схилах Центрального Великого Кавказу (район Казбегі, Грузія). Український ботанічний журнал, 78(3): 163-175 [In English].
\end{abstract}

Факультет природничих наук та медицини, Інститут екології, Державний університет Іллі, пр. К. Чолокашвілі 3/5, Тбілісі 0162, Грузія: Т. Джолохава, О. Абдаладзе, Х. Гігаурі. Факультет точних і природничих наук, Тбіліський державний університет ім. Іване Джавахішвілі, пр. І. Чавчавадзе, 3, Тбілісі 0179, Грузія: Т. Джолохава. Відділ родючості грунтів, Науково-дослідний центр сільського господарства, Міністерство охорони навколишнього середовища та сільського господарства Грузії, пр. марш. Геловані 6, Тбілісі 0160, Грузія: Т. Джолохава, Інститут етнобіології та соціальної екології, Державний університет Іллі, пр. К. Чолокашвілі 3/5, Тбілісі 0162, Грузія: 3. Кіквідзе. Інститут ботаніки, Державний університет Іллі, вул. Ботанічна 1, Тбілісі 0105, Грузія: 3. Кіквідзе. Факультет управління, Грузинський інститут громадських справ, вул. Ієтіма Гурджі 9, Тбілісі 0105, Грузія: Х. Гігаурі

Реферат. Альпійські екосистеми є особливо чутливими до кліматичних змін, що впливають на взаємозв'язки між льодовиками, сніговим покривом, рослинністю та грунтами. Метою нашого дослідження було з'ясувати вплив змін факторів абіотичного середовища на властивості грунту і розподіл видів рослин на регіональному та місцевому рівнях. Відбирали зразки грунту та аналізували рослинність уздовж двох трансект, розташованих на схилах протилежного аспекту (півночі та півдня), від альпійсько-нівального екотону до снігової лінії (Центральний Великий Кавказ, район Казбегі, Грузія). Вимірювали температури грунту та нахилу схилу. Для з'ясування зв'язків між абіотичними факторами, властивостями грунту та розподілом видів рослин за градієнтами використовували методи багатовимірної ординації. Нами було встановлено, що краще вирішеними були моделі ординації при використанні властивостей грунту як змінних середовища замість абіотичних факторів, таких як висота, нахил та аспект схилу. Кислотність грунту та вміст доступного для рослин калію виявились найкращими прогностичними показниками розподілу видів рослин у цих оселищах. Отже, моделі, що враховують роль грунтів як посередника між абіотичним середовищем і рослинністю, можуть більш точно описати розподіл видів рослин на місцевому та регіональному рівнях. Це $є$ потенційно важливим уточненням під час проведення моніторингу наслідків впливу змін клімату на рослинність, принаймні у високогірних екосистемах.

Ключові слова: абіотичні змінні, видовий склад, властивості грунту, градієнт висоти, субнівально-нівальна зона 